\section{Some like it hot}

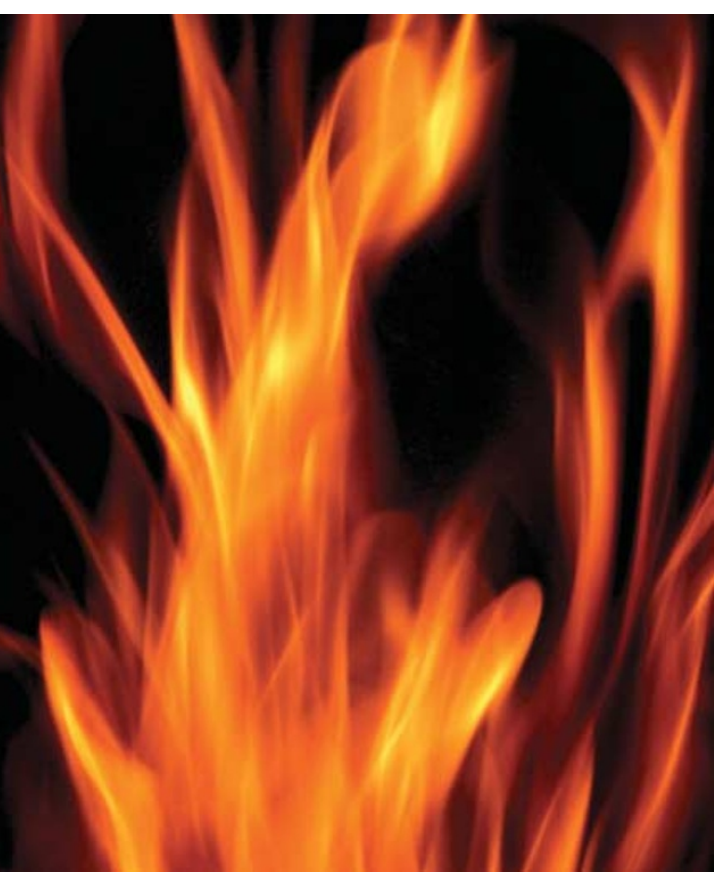

As nitrogen availability is often the limiting factor to growth and biomass production, nitrogen fixation is one of the most important biological processes on Earth. Now, new research has revealed the reasons behind the differences in the global distribution of heterocystous and non-heterocystous nitrogen-fixing cyanobacteria.

Heterocystous cyanobacteria are believed to be better adapted for $\mathrm{N}_{2}$ fixation than nonheterocystous cyanobacteria - because the heterocysts (differentiated cells enveloped by a glycolipid layer) are thought to protect nitrogenase from inhibition by molecular oxygen. Despite this however, the dominant $\mathrm{N}_{2}$-fixing cyanobacteria in the tropical oceans are the non-heterocystous Trichodesmium spp. Indeed, there are significant differences in the global distribution of hetercystous and nonheterocystous cyanobacteria - heterocystous cyanobacteria are the dominant species in freshwater lakes and brackish environments (such as the Baltic Sea), but Trichodesmium spp. are dominant in the tropical oceans, yet unsuccessful in temperate or polar oceans.

Now, reporting in Nature, Staal et al. have provided answers to two important questions regarding these differences in distribution; namely, why are free-living heterocystous cyanobacteria not the dominant $\mathrm{N}_{2}$-fixing organism in the tropical oceans, and why are Trichodesmium spp. not able to thrive in temperate and polar regions, and in freshwater and brackish environments?

Using an acetylene reduction assay, the authors compared nitrogenase activity in the heterocystous Nodularia spumigenia strain CCY 9414 and Anabaena sp. strain CCY 9901 with the nitrogenase activity in Trichodesmium sp. strain IMS101, and showed that $\mathrm{N}_{2}$ fixation in the dark in both heterocysts and diazocytes (the specialized $\mathrm{N}_{2}$-fixing cells of Trichodesmium which do not have a glycolipid layer) is limited by $\mathrm{O}_{2}$ diffusion. In the dark, respiratory ATP production limits $\mathrm{N}_{2}$ fixation due to a limited influx of $\mathrm{O}_{2}$ to the heterocyst or diazocyte. However, in the light, ATP production by the photosynthetic electron transport chain ensures that ATP availability is not a limiting factor.

The authors also examined the effect of increased temperature on the gas flux and enzyme potential in the $\mathrm{N}_{2}$-fixing cells. Although the concentration of dissolved $\mathrm{O}_{2}$ decreases with increased temperatures, the gas diffusion coefficient increases - resulting in a net increase in the gas flux into the $\mathrm{N}_{2}$-fixing cells. However, the enzyme potential increases

\section{PROTOZOAN PARASITES}

\section{Trypanosome flagellum}

The single flagellum of Trypanosoma brucei has been unveiled as a key player in determining cell polarity, division, size and shape.

The flagellum, an appendage whose structure is conserved from protists to mammals, extends from the cell body. It is assembled by adding subunits to the distal tip. However, the flagellum lacks the machinery for protein synthesis and a transport system for subunits synthesized in the cell body is needed. A specialized system, known as intraflagellar transport (IFT), delivers 'rafts' of proteins that are synthesised in the cell body to the flagella tip, and can also transport proteins from the flagellum back to the cell body. Motor proteins drive movement of rafts, which contain additional IFT proteins known as cargoes. Defects in IFT prevent flagellum formation in most eukaryotes tested so far. In new research published in the $E M B O$ Journal RNAi and cell biology were combined to probe the function of the trypanosome flagellum.

Kohl et al. mined T. brucei genome sequences for putative IFT homologues. From several homologues identified, two genes were selected. One gene encodes a putative cargo protein whilst the other gene encodes a putative motor protein. By coupling gene silencing using RNAi with microscopy, Kohl et al. showed that separate silencing of either the putative motor or cargo homologue resulted in progressive flagellum shortening. A shortened flagellum is initially produced because RNAi results in a dwindling pool of IFT proteins, which in turn reduces transport of the proteins needed for flagellum assembly. Eventually daughter cells had no flagellum. So, IFT is functional in T. brucei and is required for flagellum assembly and for control of flagellum length. Mutant cells lacking a flagellum lost polarity, as demonstrated by mislocalization of

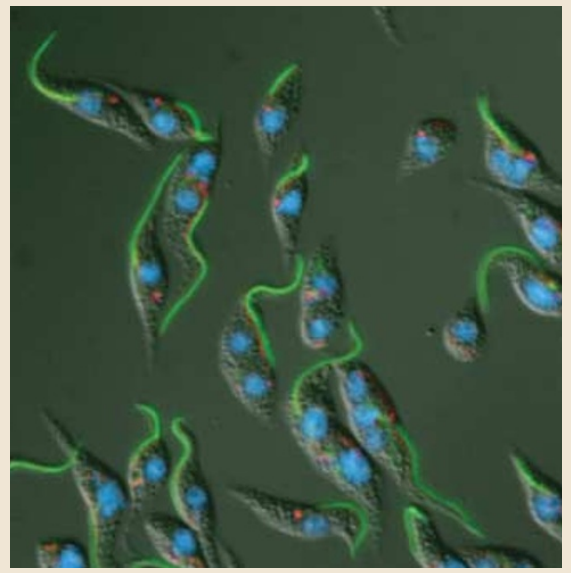

Image shows a mixture of trypanosomes with full-length, shortened or no flagellum (stained green), basal body (stained red) and DNA (stained blue) viewed by differential interference contrast microscopy. Courtesy of Linda Kohl and Philippe Bastin (Laboratoire de Biophysique, Muséum National d'Histoire Naturelle, Paris, France).

clathrin, a typical cell organzation marker. These mutants also lost their distinctive shape and had no clear anterior or posterior end. So, both cell polarity and internal organization were lost when flagella assembly was disrupted.

During induction of RNAi, mutants not only produced a shortened flagellum but 
faster than the gas flux, so with incerasing temperatures, nitrogenase activity becomes substrate limited, particularly in those cyanobacteria with an efficient diffusion barrier — heterocystous cyanobacteria. In addition, by modelling the influx of $\mathrm{O}_{2}$ to both heterocysts and diazocytes, Staal et al. calculated that $\mathrm{O}_{2}$ flux increases by approximately $25 \%$ when the salinity is decreased from 35 (sea water) to 0 (fresh water) - thereby, highlighting the need for an efficient diffusion barrier, such as the glycolipid envelope of the heterocyst, in freshwater conditions.

So, in freshwater conditions, the glycolipid envelope of the heterocyst provides a selective advantage over non-heterocystous cyanobacteria - explaining the absence of Trichodesmium from freshwater seas. But, in pelagic environments at high temperatures, the glycolipid envelope is a disadvantage explaining the dominance of Trichodesmium in the tropical oceans.

(1) References and links

ORIGINAL RESEARCH PAPER Staal, M., Meysman, F. J. R. \&

Stal, L. J. Temperature excludes $\mathrm{N}_{2}$-fixing heterocystous

cyanobacteria in the tropical oceans. Nature 425, 504-507 (2003)

WEB SITE

The Netherlands Institute of Ecology:

http://www.nioo.knaw.nl/CEME/MM/index.htm

were smaller than wild-type cells. The length of the flagellum correlated with cell size - the shorter the flagellum, the smaller the daughter cell. Before cell division, a new flagellum is assembled alongside the old flagellum. By monitoring mutants during induction of RNAi the authors deduced that the tip of the newly synthesised flagellum defines the site of cytokinesis. Alongside the flagellum in the cytoplasm there is a flagellar attachment zone (FAZ) which also replicates before cell division. Mutant trypanosomes which completely lack flagella still have a FAZ but its shape is shortened and irregular. Since these cells can still divide, the FAZ is the best candidate for a marker of cytokinesis.

During the life cycle of T. brucei, there are several morphologically distinct cell-types. It's possible that regulating IFT — and therefore regulating flagellum length could be used to modulate parasite differentiation.

\section{(2) References and links} ORIGINAL RESEARCH PAPER Kohl et al. Novel roles for the flagellum in cell morphogenesis and cytokinesis of trypanosomes. EMBO J. 22, 5336-5345 (2003)

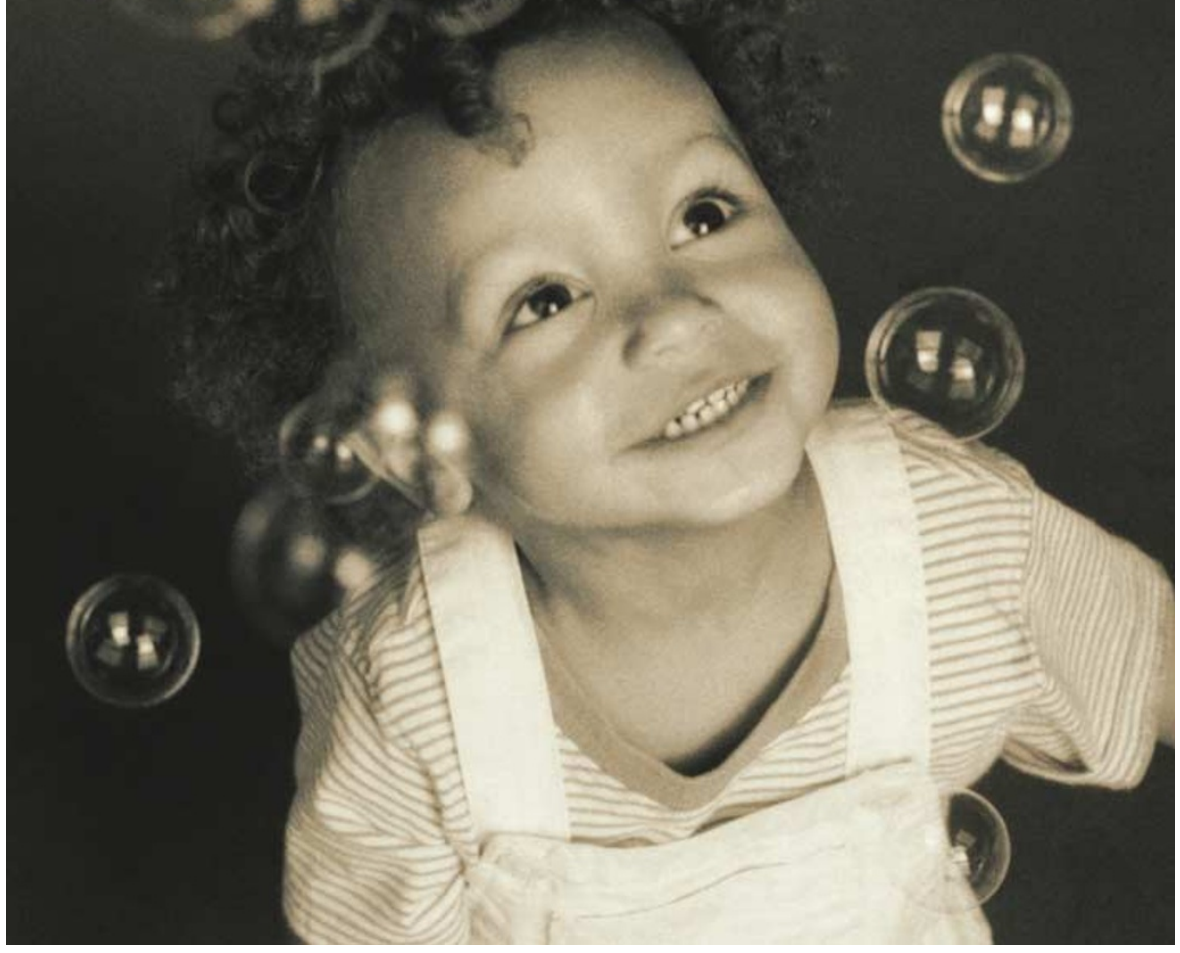

BACTERIAL PHYSIOLOGY

\section{Bacterial secretion into vesicles}

Five distinct secretion pathways are used to transport proteins across the Gram-negative bacterial double membrane. This is in addition to three transport routes across the inner membrane. Even so, Escherichia coli, the beststudied bacterium, can still spring a surprise on microbiologists. Reporting in Cell, Wai et al. describe a new mechanism of secretion in which a toxin is exported in vesicles. Vesicle or bleb formation is nothing new - but this is the first time vesicle-mediated secretion has been given clear physiological relevance.

Common laboratory strains of E. coli can produce ClyA, a cytotoxin that is secreted into the bacterial periplasm but acts on mammalian cells. How does the toxin access its target site? Using immunofluorescence, Wai et al. showed that ClyA is surface-exposed. Closer examination with electron microscopy and atomic force microscopy revealed a multitude of small outer-membrane vesicles (OMVs) approximately 50-200 nM in diameter surrounding the bacteria. Analysing the protein content of these vesicles proved that they were derived from the outer membrane. Strains of E. coli that do not produce ClyA still produced OMVs. Reassuringly, pathogenic E. coli and Salmonella enterica serovar Typhi strains that produce ClyA also localized the toxin into OMVs, so this process isn't restricted to laboratory-adapted strains. The OMVs typically had ring-like pores, which were shown to be assemblies of ClyA protein.

Toxicity assays showed that ClyA present in OMVs was at least 8 times more toxic than purified ClyA. This striking result prompted the authors to examine ClyA multimerization, and cross-linking experiments confirmed that periplasmic ClyA is monomeric compared with multimeric ClyA present in OMVs. Further, removing the disulphide-bond oxidation machinery from E.coli dramatically increased the action of ClyA. The authors couldn't detect proteins that participate in disulphide-bond formation in OMVs containing ClyA, raising the possibility that the redox status of ClyA contributes to oligomerization and toxicity. OMVs seem to contain a subset of periplasmic proteins, which means that E.coli could have a crude protein-sorting mechanism that selects which proteins are exported in OMVs.

OMVs are formed by a whole variety of bacterial species, including commensal Bacteroides species and pathogenic Neisseria species. If bacteria can sort periplasmic proteins into vesicles, the challenge will be to find out how sorting is accomplished, how it is regulated and whether this process can be used as a model for eukaryotic vesicle trafficking.

\section{Susan Jones}

\section{6) References and links}

ORIGINAL RESEARCH PAPER Wai et al. Vesicle-mediated export and assembly of

pore-forming oligomers of the enterobacterial

ClyA cytotoxin. Cell 115, 25-35 (2003)

FURTHER READING Miller, S. I. et al. Bacterial vesicle

formation as a mechanism of protein transfer to animals. Cell 115, 2-3 (2003)

WEB SITE

Bernt-Eric Uhlin's laboratory:

http://www.molbiol.umu.se/forskning/uhlin/ 